Emmanuel Housset

Université de Caen Normandie

Identité et Subjectivité

\title{
La crise de la laïcité et la philosophie
}

\section{Quelle place pour une interrogation philosophique?}

La laïcité est en crise aujourd'hui et cela signifie d'abord qu'elle n'est plus « évidente », qu'elle ne fait plus l'objet d'un accord tacite, qu'elle n'est plus un de ces principes inavouables qui sont aux fondements d'une communauté, pour parler avec Blanchot ${ }^{1}$. On peut bien sûr se demander s'il y a eu dans l'histoire un moment où la laïcité allait vraiment de soi, ou bien s'il ne s'agit pas là d'un passé mythique, voire d'une fiction dans laquelle les personnages de Peppone et de Don Camillo se chamaillaient tout en visant le bien commun. Quoi qu'il en soit, même si l'histoire de la laïcité est l'histoire de ses crises, il n'en demeure pas moins que cette crise dans l'humanité européenne prend aujourd'hui une forme très particulière qui est liée à l'Islam, même si bien évidemment elle ne se résume pas à lui, même si on ne peut pas considérer l'Islam comme la cause efficiente de cette crise, mais plutôt comme son catalyseur. Autrement dit, par des difficultés attribuées à l'Islam, la laïcité est redevenue une question, mais ce n'est pas parce qu'il y a la conscience d'une crise que la crise est identifiée comme telle. C'est une chose de dire qu'il y a une crise, c'en est une autre de pouvoir élucider quels sont les véritables constituants de cette crise et ce qui se joue dans cette crise.

Il est alors possible de se demander quelle parole sur la laïcité le philosophe en tant que tel peut tenir, alors que cette question semble relever de la sociologie, de l'histoire et du droit. Afin de donner toute sa force à la question, il convient de ne pas prendre le terme de philosophie dans un sens totalement indéterminé. La philosophie n'est pas un bavardage mondain de personnalités notoires, mais, d'une manière plus discrète et profonde, elle est une science rigoureuse qui cherche à élucider les premiers principes de l'être. Avant de décrire l'espace social dans lequel le problème de la laïcité en vient à se poser, son projet est, par exemple, de conduire à la clarté l'essence même de tout espace. La possibilité même pour le philosophe de dire, en tant que philosophe (et non en tant qu'il est aussi citoyen ou un individu pris dans des jeux de forces), quelque chose de sensé sur la laïcité ne va nullement de soi. Pourtant, dans son souci d'élucidation des concepts, le philosophe peut déjà rappeler, en faisant référence à l'étymologie, qu'une crise n'est pas seulement un moment où le sens s'efface, mais est également un temps de discernement, de jugement, et c'est pourquoi si la période actuelle est inquiétante, elle est également très riche en horizons de réflexion. C'est peut-être déjà trop s'avancer, mais il semble que les éléments récents indiquent que nous sommes en un lieu de partage des eaux : soit la crise de la laïcité va conduire vers un pragmatisme et un relativisme proposant des réponses momentanées, mais sans véritable analyse, soit elle va être l'occasion d'un retour de l'humanité vers elle-même afin de redéfinir les rapports du religieux et du politique. Ainsi avant d'aborder cette question

\footnotetext{
${ }^{1}$ Maurice Blanchot, La communauté inavouable, Paris, Minuit, 1983.
} 
de la laïcité avec un regard de philosophe, il n'était pas inutile de rappeler que la place du philosophe est par essence complexe. Il n'est pas « dans » le monde et ne peut pas partir de la description empirique des mille et une formes de la crise de la laïcité aujourd'hui, s'il ne veut pas se perdre dans l'indéfini des situations. En ce sens-là il n'est pas non plus celui qui jette de l'huile sur le feu en attaquant les religions ou la vie politique en général et il est plutôt celui qui dépassionne les débats afin de déployer une réflexion intempestive au sens de Nietzsche. Il n'est pas non plus celui qui est hors du monde, simple spectateur impartial, car il est toujours déjà pris par le monde selon un sens de l'engagement développé par l'existentialisme. Le philosophe est donc aussi celui qui doit tenter de répondre du sens d'une situation historique, et en ce qui concerne l'objet de cette étude il s'agit bien de répondre au nihilisme des actes terroristes. Encore une fois, sans se prendre pour un historien ou un sociologue, le philosophe se doit de répondre au nihilisme et la crise de la laïcité donne à penser une des formes du nihilisme contemporain.

Pour le philosophe se pose ensuite une question de méthode s'il ne veut pas fonder sa réflexion sur un sol instable. En effet, il ne peut pas partir d'emblée de la question des rapports possibles entre laïcité et religion, car il s'agit de deux idées générales et abstraites dont on ne peut pas se faire facilement une idée claire et simple. «La » religion, cela n'existe pas, et même tout simplement parler « des » religions cela peut sembler très abusif, car il n'y a parfois aucun élément commun qui permette de rassembler sous une même catégorie des croyances et des pratiques totalement différentes. Y-a-t-il vraiment une essence de la religion au-delà de certains regroupements purement empiriques ? Il semble difficile de rassembler le Vaudou et le Judaïsme autour de quelques points communs minimaux comme un certain rapport à une transcendance ou au sacré. Bien évidemment, il existe de nombreuses histoires des religions, des encyclopédies des religions, mais pour le philosophe il ne va pas de soi qu'il y ait là un « objet » déterminé. On peut ajouter que les polythéismes ne se ressemblent pas et les monothéismes non plus. Comme le souligne Rémi Brague, parler de monothéisme ou de trois monothéismes n'apporte aucun éclaircissement sur ces trois religions ${ }^{2}$. En outre il peut y avoir des religions sans dieux, sans Eglise, comme il y a du sacré sans religion. Bref, dans sa méthode, le philosophe ne peut pas faire l'économie de ce temps de l'aporie, de ce moment pendant lequel tout ce que l'on imaginait ternir fermement s'évapore. La plupart du temps on ne sait pas bien de quoi on parle quand on évoque «la religion » et du coup il est possible de soupçonner les défenseurs de la religion comme les contempteurs de toute forme de religiosité d'inventer ce qu'ils défendent ou refusent. Un christianisme inventé est bien plus simple à défaire que le christianisme réel. La difficulté de cette production de phantasmes, qui répond souvent à des besoins de domination bien précis, est qu'elle noie la question au lieu de permettre de la construire. On comprend que certains se réfugient dans le confort de l'anticatholicisme, moins risqué que l'anti-islamisme, mais ce ne sont que des sophistes, ces doubles monstrueux du philosophe décrits par Platon, qui se soucient seulement de plaire et de ridiculiser l'adversaire dans le mépris de la vérité.

Une difficulté analogue se pose avec le terme de laïcité qui est très opaque et qui peut conduire à absolutiser certaines formes historiques relatives, comme par exemple « la laïcité à la française » par rapport à la soi-disant « laïcité boiteuse à l'allemande ». La laïcité n'est pas une valeur éternelle, mais bien une idée historique assez récente qui a subi de très nombreuses variations. Une fois encore l'essence semble échapper au regard réflexif et le « bien connu » est « mal connu »; c'est pourquoi il ne suffit pas de prononcer le mot de laïcité pour avoir fait de la philosophie politique. Face à une telle idée obscure et confuse, la tâche du philosophe n'est pas d'établir les faits, d'autres le font bien mieux que lui, mais il peut faire de la laïcité une expérience de pensée en cherchant à élucider la séparation du religieux et du politique, en s'interrogeant sur la signification d'une neutralité du politique, ce qui ne conduit pas nécessairement à une affirmation d'athéisme. Il faut bien reconnaître que le projet politique actuel d'enseigner la laïcité est en lui-même symptomatique : cela signifie que notre culture ne transmet pas d'elle-même cette valeur, puisqu'elle doit être enseignée, et donc qu'il s'agit d'une valeur dévalorisée.

\footnotetext{
${ }^{2}$ Rémi Brague, Du Dieu des chrétiens et d'un ou deux autres, Paris, Flammarion, 2009, p. 16 et sv.
} 
En outre, il est toujours à craindre que les cours de laïcité se résument parfois à de l'endoctrinement, or comme disait déjà saint Augustin dans le traité Du maître, qui serait assez sot pour envoyer ses enfants à l'école afin qu'ils apprennent ce que le maître pense et non des sciences. On peut donc avancer que ce qui se trouve en cause dans la crise actuelle de la laïcité, ce n'est pas seulement l'affirmation de la laïcité, le fait qu'elle puisse être contestée comme principe de vie commune, mais c'est également l'essence même de la laïcité. Pour prendre un vocabulaire plus philosophique, on a peut-être une conscience plus aigüe aujourd'hui de la tension entre l'idée universelle de laïcité et la facticité de sa réalisation historique dans une situation donnée. C'est pour cela que la compréhension française de la laïcité ne peut pas être identique à sa compréhension allemande, bien plus souple, car liée à l'adage issu de la Réforme, cujus regio, ejus religio. Dès lors, si la crise de la laïcité semble participer à la crise actuelle des valeurs, la tâche propre de la philosophie est sans doute de rechercher une troisième voie entre la tentation utilitariste de réduire la laïcité à un compromis contingent étranger à toute mesure idéale et la tentation idéaliste d'affirmer des valeurs universelles indépendamment de tout rapport au monde et de tout pragmatisme dans l'action.

\section{Laïcité et neutralité}

La question est de savoir si le principe républicain de laïcité est capable de rassembler une multitude d'individus, avec des cultures et des religions différentes, en un peuple uni par des règles de droit. D'une certaine manière, la mise en œuvre de la République est peut-être plus complexe aujourd'hui qu'auparavant, dans la mesure où la diversité ne tient pas seulement à la langue ou à la culture, mais également à la religion. Les conflits ne sont plus seulement internes à la tradition biblique, mais ont lieu entre toutes les religions ainsi qu'entre les religions et l'athéisme. Certes le problème n'est pas nouveau, et il suffit de se rappeler les guerres de religion, mais la situation actuelle possède tout de même des dimensions irréductibles au passé. La question est de savoir comment vivre ensemble dans le respect des différentes religions, comme de l'absence de religion, sans renoncer à ce qui fait la culture européenne, au sens non-géographique du terme, à savoir l'idée d'une culture issue de la raison libre, comme l'a développée Husserl dans une célèbre conférence de 1936 La crise de l'humanité européenne et la philosophie ${ }^{3}$. Il y a là une tension interne, un conflit de valeurs, entre deux compréhensions de la liberté. Dans l'attitude religieuse la liberté est un consentement éclairé par la raison à un bien qui est reçu par l'homme, alors que dans la compréhension contemporaine l'absolue dignité de la personne se fonde sur un idéal d'autonomie, c'est-à-dire sur une capacité d'auto-législation du sujet libre ${ }^{4}$. Il ne s'agit donc pas d'une simple opposition brutale entre foi et raison et le mérite de la crise de la laïcité est de mettre en lumière une aporie théorique, pratique et politique sur l'exercice de la liberté. Il serait sans doute injuste de réprimer toute expression publique des convictions religieuses, mais alors comment faire en sorte que cette expression ne soit pas insupportable aux autres et ne soit pas contraire aux valeurs de la République?

Pour le philosophe, la crise de la laïcité est une crise des fondements de la vie sociale, même s'il n'ignore pas que cette crise a également d'autres causes, des causes économiques, socio-historiques, démographiques, politiques, etc. Encore une fois il y a là une question de méthode : la tâche du philosophe n'est pas de donner son avis sur ces causalités sur lesquelles il est incompétent ou sur lesquelles il est au mieux un amateur éclairé, mais il doit les mettre entre parenthèses, c'est-à-dire suspendre son jugement sur elles, pour se concentrer sur ce qui relève de sa propre capacité, à savoir l'idée de laïcité comme idée, voire comme idéal rationnel. L'idée de laïcité, dans sa capacité à être partagée par tous, semble une idée rationnelle dont la naissance est assez récente et qui pourtant s'est

\footnotetext{
${ }^{3}$ Edmund Husserl, La crise de l'humanité européenne et la philosophie, trad. Paul Ricœur, Paris, Aubier, 1987.

${ }^{4}$ Chez certains penseurs comme Durkheim ou Mauss, c'est la personne humaine elle-même qui devient alors sacrée. Voir Hans Joas, Comment la personne est devenue sacrée, trad. Jean-Marc Tétaz, Genève, Labor et Fides, 2016, p. 92.
} 
effondrée avec les autres valeurs, comme la liberté, l'égalité et la fraternité. La fonction propre du philosophe, sa responsabilité sociale, est alors sans doute de tenter de reprendre une telle idée, de lui redonner vie. Ce n'est pas au philosophe de comparer la laïcité à la française et la laïcité à l'allemande et de suivre leur évolution respective, mais il peut tenter de faire accéder une telle idée à une plus grande clarté, de l'arracher à sa seule détermination négative de l'anticléricalisme, de l'anti-catholicisme ou de l'anti-islamisme. De façon classique, on accuse une telle réflexion d'abstraction et d'être inefficace du point de vue politique et on voit bien l'écart entre l'idée générale de laïcité et sa mise en œuvre dans des décrets nationaux ou locaux dans lesquels elle semble n'être plus que le résultat de groupes de pression et non une règle de justice. D'une part, le rôle du philosophe n'est pas de dire ce qui doit être fait en politique ; comme le montrait déjà Platon, il invite le roi à se faire philosophe, mais ne devient pas roi lui-même. Il n'y a rien de pire qu'un philosophe voulant se faire roi. Ainsi, d'autre part, ce qui relève de sa compétence, c'est d'éclairer l'idée de laïcité pour que l'homme politique détienne un concept le plus précis possible à partir duquel ce sera à lui de déterminer des moyens. Dans ce domaine comme dans d'autres le grand danger est le scepticisme, cette haine de la raison qui conduit à dire qu'aucune clarté ne peut être obtenue et que la justice n'existe pas. C'est une façon de trancher le problème de la laïcité sans le dénouer.

Jean-Luc Marion dans son ouvrage récent ${ }^{5}$, défend que le seul sens valable de la laïcité est la neutralité. L'Etat sous toutes ses formes doit rester «neutre », précisément parce que les personnes qui constituent la société ne le sont pas. Il va de soi que l'athéisme n'est pas une position neutre, pas plus que l'agnosticisme. Ainsi le médecin comme le fonctionnaire se doivent de demeurer neutres, c'est-àdire de suspendre toute déclaration sur leurs convictions personnelles pendant leur travail. La seule limite à cette neutralité de l'Etat est négative, c'est-à-dire interdire tout ce qui porte atteinte à la dignité de la personne humaine. Cela dit, il est également clair qu'il suffit d'avancer une telle affirmation pour ouvrir des problèmes sans fond, car il n'y a pas d'évidence commune sur cette atteinte à la dignité de la personne humaine. Là aussi il faut renoncer aux abstractions et au phantasme d'une action politique "pure », n'obéissant qu'à des principes rationnels, sans aucun compromis avec la réalité. Tout ce qui relève de l'action, et a fortiori l'action politique dans une République, est la recherche d'un équilibre du moment, de ce qui est possible et réalisable dans une situation donnée, sans renoncer aux principes. Une bonne loi, celle qui ne reste pas lettre morte ou qui n'est pas violente, est une loi d'équilibre qui manifeste ce que les penseurs grecs et médiévaux nommaient la «prudence» et qui n'a rien à voir avec le sens contemporain de simple habileté utilitariste. Ainsi la paix suppose la neutralité de l'Etat, à savoir une

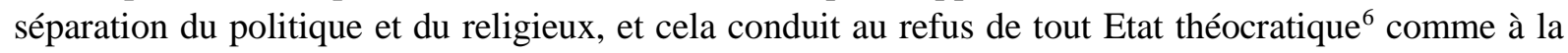
remise en cause du principe défendu par Montaigne de prendre la religion de son pays. La tolérance est également une idée historique et elle a changé aujourd'hui de nature, elle demande une autre souplesse, bien plus difficile, celle de la cohabitation des différentes religions, ainsi que des athées et des croyants. Dès lors, ce principe de neutralité formule aussi une exigence à l'égard de la religion, à savoir qu'elle ne cherche pas à se transformer en groupe de pression, en force politique, sans renoncer à expliquer et à convaincre. La neutralité n'est donc pas une simple indifférence, ni un simple équilibre de circonstance, mais un principe a priori de la vie politique, condition de possibilité d'une unité politique dans la diversité des convictions.

\section{La laïcité une idée neuve en Europe}

Il n'est pas interdit de penser qu'au-delà de la seule situation française l'idée d'une telle « séparation » soit encore une idée neuve en Europe et qu'elle ait pour vocation de s'étendre à la totalité du monde, de manière à ce que le monde ne se réduise pas à sa division en zones d'influence des

\footnotetext{
${ }^{5}$ Jean-Luc Marion, Brève apologie pour un moment catholique, Paris, Grasset, 2017, p. 43.

${ }^{6}$ Un Etat religieux n'est pas nécessairement un Etat théocratique comme tentera de le dire Emmanuel Levinas de l'Etat d'Israël dans Difficile liberté, Le livre de proche, 2006, p. 328. De même une religion d'Etat comme au Royaume-Uni n'est pas incompatible avec la laïcité.
} 
religions, avec pour conséquence la violence de la majorité sur les minorités. En effet, dire que la laïcité est une idée neuve, ce n'est pas nier son point de départ historique, mais c'est reconnaître que cette idée a un avenir et qu'elle est loin d'avoir fini son histoire et que c'est peut-être à cette condition qu'elle est la seule à pouvoir désamorcer la dimension religieuse des guerres politico-religieuses. On pourrait sans doute écrire une histoire des religions à partir de leur capacité à intégrer l'idéal de la laïcité, mais il serait certainement également envisageable de construire une histoire politique à partir de la même mesure, surtout quand on pense aux millions de morts des athéismes du XXème siècle. Autrement dit, il y a autant de difficulté à incorporer cette idée de laïcité dans le corps politique que dans les communautés de croyants. Une fois libéré du laïcisme, d'une certaine manière la maladie infantile de la laïcité, il est possible d'envisager une telle nouvelle histoire. Or cette histoire est aussi structurée par un avenir et un avenir qui nous appartient : serons-nous, en tant que citoyens et en tant que personnes pouvant avoir des convictions religieuses, capables de porter cette idée ? Dans cette perspective, il ne suffit pas d'avancer qu'il ne devrait pas y avoir de religion d'Etat, ni de reprendre toute l'histoire des conflits entre le politique et le religieux, encore faut-il que cette idée de laïcité perde de son abstraction afin de devenir un principe effectif de réflexion et d'action. Il faut bien reconnaître que ce principe est essentiel pour l'Etat dans la mesure où la reconnaissance de la diversité des croyances est ce qui sert de garde-fou contre toute tentation d'une dérive totalitaire dans l'affirmation brutale de l'athéisme ou d'une religion. Comme l'a bien montré Jean-Luc Marion dans l'ouvrage déjà cité, ce principe n'est pas simplement une précaution contre les dérives de la religion, il est également essentiel à la religion elle-même en lui permettant d'éviter toute confusion du spirituel et du temporel. Ainsi, positivement comprise, la laïcité c'est la liberté de conscience religieuse, la liberté de se convertir, tout autant que la liberté de ne pas avoir de religion. Jean-Luc Marion défend alors l'idée que la crise actuelle n'est pas celle de la fin d'une histoire et qu'elle est sans doute la manifestation d'une nouvelle époque qui commence, une époque dans laquelle la confusion entre le politique et le religieux peu à peu se dissipe, dans laquelle l'idée de laïcité est toujours mieux mise en lumière ${ }^{7}$.

Le philosophe est celui qui fait de la responsabilité à l'égard du sens du monde la forme même de sa vie, et en cela il ne peut comprendre l'échec de la laïcité que comme son échec : il n'a pas élucidé avec assez de sérieux l'idée de laïcité de manière à rendre possible une réflexion du corps politique sur lui-même et des religions sur elles-mêmes. Cela dit, pour le philosophe, il ne s'agit pas d'exclure que sur ce point l'humanité puisse échouer, que ce soit là l'une des manifestations tragiques de sa finitude. Autrement dit, la laïcité est une idée vulnérable, une idée qui peut s'abîmer ou même disparaître au sens où elle ne serait plus portée par personne. C'est une idée qui a la valeur d'un impératif, mais encore fautil vouloir cet impératif en évitant l'indifférence ou l'affirmation somnambulique de ses convictions. On s'est souvent moqué du dialogue inter-religieux, l'accusant parfois arbitrairement de dérive syncrétiste, ou d'être une exigence purement chrétienne, et pourtant c'est bien de lui que dépendra en partie l'incorporation de l'idée de laïcité. La laïcité n'est donc pas une leçon d'humanisme de la République à la religion, ce qui serait absurde, mais elle est une idée rationnel qui n'est d'aucun pays, d'aucun peuple, même si elle est bien née quelque part, et dont la République elle-même n'est pas propriétaire.

\section{Universalité et singularité}

La foi rationnelle du philosophe est que seul l'universel peut sauver l'humanité, mais alors comment concilier l'universalité du principe de laïcité et la singularité des situations sociales et historiques ? Le véritable universel, celui qui n'est pas une simple abstraction, n'est pas celui qui détruit le particulier, mais celui qui le révèle. Dès lors, idéalement, la laïcité ne devrait pas abolir la particularité de chaque religion, mais mettre au jour son style propre en lui donnant une place dans l'espace commun. Cela dit il faut bien reconnaître que la laïcité n'est pas un simple relativisme qui accepte tout et qu'elle exerce un rôle normatif sur les religions. On pourrait alors avancer que ce que la laïcité en tant

\footnotetext{
${ }^{7}$ Jean-Luc Marion, Brève apologie pour un moment catholique, Paris, Grasset, 2017, p. 68.
} 
qu'universel met en cause, c'est le particulier qui n'est qu'un particularisme, qui est un processus identitaire d'exclusion. Encore faut-il être capable de faire clairement la part de l'un et la part de l'autre, ce qui ne va pas du tout de soi. On ne peut que constater la difficulté qu'il y a à porter cet universel, à l'expliquer à tous ceux pour lesquels il n'a aucun caractère d'évidence car il n'appartient pas d'emblée à leur tradition. Bien des historiens rappellent que si on entend par laïcité la séparation du spirituel et du temporel, la laïcité est une idée d'origine chrétienne ${ }^{8}$, même si le christianisme est loin d'avoir été toujours fidèle à cette idée et même si aujourd'hui encore la tentation de la confusion des ordres est grande, comme, par exemple, dans le christianisme orthodoxe.

Aucune idée n'a d'incarnation historique parfaite et il serait dangereux de considérer la loi française de séparation de 1905 comme la seule forme possible de séparation du spirituel et du temporel. Il n'y a donc pas de modèle français en matière de laïcité et la reconnaissance par un Etat d'une religion n'est pas nécessairement un renoncement à la laïcité. Il y a d'autres manières possibles de vivre la laïcité que celle liée à l'histoire française ; chaque pays a son histoire et c'est donc en fonction de son historicité propre que la politique doit être créatrice d'une forme spécifique de laïcité. Ici la tâche du philosophe est de dénoncer toute tentation d'une absolutisation d'une figure historique relative et contingente. En conséquence il est possible de défendre l'idée que si la laïcité est un ethos qui est né en Europe avec le christianisme et qui a pris une forme historique particulière avec la loi de 1905 en France, il s'agit d'un ethos qui est réappropriable en droit par toutes les traditions. Autrement dit, l'idée d'une neutralité confessionnelle de l'Etat, de la liberté religieuse comme celle de n'avoir pas de religion, l'autonomie de la conscience personnelle par rapport à tout pouvoir, l'exigence d'une absence de limite du pouvoir critique de la raison ${ }^{9}$ constituent bien un ethos qui peut devenir commun, mais qui doit trouver une traduction particulière dans chaque tradition. C'est là qu'il convient de manifester la plus grande prudence afin de ne pas tomber dans un européannocentrisme imposant une figure particulière et être attentif à la créativité de chaque corps politique, pour que cet ethos prenne une forme historiquement possible, une forme qui ne se fige pas, qui est en quelque sorte toujours en formation. En outre, une telle laïcité n'est en rien incompatible avec le fait que certains citoyens vivent une obéissance religieuse et même contestent certaines lois politiques au nom de convictions religieuses. Sans pouvoir déterminer ce qu'il faut faire, la philosophie peut chercher à clarifier au moins la forme d'une vraie laïcité en soulignant notamment que si l'Eglise peut être une force de proposition et de contestation, elle met en cause la laïcité quand elle veut se faire pouvoir politique. Ce n'est pas la même chose pour une loi d'avoir une « inspiration » religieuse et de trouver sa légitimité dans la religion. Formellement la laïcité est alors cette indépendance de la communauté politique et de la communauté religieuse, chacune ne devant trouver sa légitimité qu'en elle-même.

La laïcité est donc un idéal qu'il s'agit de développer hors Europe, mais qui même en France doit toujours à nouveau trouver son expression historique. Il y a peut-être un problème avec l'Islam, qui n'est pas nécessairement qu'un problème de l'Islam, et on peut avancer, au moins au titre d'hypothèse de travail, que la mise en œuvre du principe de laïcité aujourd'hui en France n'est plus adéquate à la population française. Cette laïcité « à la française » n'est-elle pas perçue implicitement à la fois comme le projet de faire d'une religion (le christianisme, puisque c'est son origine historique) la mesure des autres, et le projet de faire de la conception de l'homme issue des Lumières, l'individu autonome, la norme de toute vie sociale? Le paradoxe actuel en France, pour ce que peut en percevoir un nonspécialiste des faits sociaux, c'est peut-être que la réussite de la sécularisation, la désacralisation de toute autorité, semble produire la révolte des laissés-pour-compte de cette laïcisation, la révolte de ceux qui se sentent sans avenir dans une société qui n'envisage que l'autonomie de l'individu. Ce passage d'un sacré commun à un sacré individuel peut expliquer la recherche dans le religieux parfois le plus aveugle de la possibilité d'appartenir à une communauté et de la possibilité de ne pas lutter seul face à la violence d'une époque. Encore une fois, ce terrain est glissant pour le philosophe, car ce n'est pas à lui d'établir

\footnotetext{
${ }^{8}$ Voir Jean-Paul Willaime, Europe et religions. Les enjeux du XXIème siècle, Paris, Fayard, 2004, p. 44 et sv.

${ }^{9}$ Je reprends là les quatre critères de Jean-Paul Willaime, op. cit., p. 46, non sans les modifier légèrement.
} 
les faits, mais au sociologue ou à l'historien, néanmoins il peut se demander quel universel a été produit par la laïcisation de la société en Europe. Il n'est pas illégitime de soupçonner qu'il s'agit d'un universel abstrait, d'une simple représentation du monde dominante, dans laquelle la communauté sociale se réduit au regroupement d'individus devant être autonomes. On est alors loin de l'universel véritable évoqué plus haut, qui au lieu de détruire le particulier lui permet de s'accomplir dans ce qu'il a de meilleur. Il n'est pas absurde de penser que l'universel abstrait, avec le nihilisme qui lui appartient, est peut-être l'une des causes du communautarisme, de l'enfermement identitaire, de l'identité comme refuge et non comme tâche à accomplir. Il ne s'agit pas de remettre en cause l'impératif formulé par Kant dans Qu'est-ce que les Lumières ?, précisément à la fin de l'époque des Lumières, celui d'être un Selbstdenker, de penser par soi-même, mais de contester son interprétation comme refus de toute autorité religieuse. On peut estimer que l'exigence kantienne s'est dévoyée en un relativisme faisant de l'individu un producteur de ses propres normes. Les violences contemporaines prennent peut-être leur source dans l'erreur d'un certain rationalisme, dans cette compréhension relativiste de l'autonomie.

Ainsi la laïcité, et c'est le point le plus difficile, est à la fois une certaine neutralité de l'Etat et l'idée que l'Etat ne se contente pas d'assurer un consensus entre les tendances du moment comme il gère les affaires économiques. Les questions spirituelles ne relèvent pas d'une « gestion », mais d'un dialogue qui seul peut rendre possible une unité des personnes et que l'Etat peut favoriser, sans l'imposer ou le diriger. C'est ainsi en favorisant la création d'un tissu social, sans se substituer à la société, qu'il peut lutter contre les dérives identitaires violentes. On est loin ici de la conception de la tolérance développée par Locke. Il ne s'agit pas d'une simple théorie libérale dans laquelle toutes les religions sont acceptées, puisqu'il n'y a pas de vérité en ce domaine, tant que le bien commun n'est pas mis en cause et que la liberté de chacun se trouve préservée. Il est en effet possible d'envisager une conception plus active de la tolérance dans laquelle le bien public dépend du dialogue entre religions et entre croyants et athées. C'est là que s'arrête le philosophe, ce qu'on lui reproche souvent, et que commence la créativité politique. Il ne peut pas dire quelles peuvent être aujourd'hui les organisations qui rendront effectives l'idée de laïcité et il peut juste mettre en évidence la nécessité d'une telle réflexion politique afin de ne pas en rester à faire coexister des altérités irréductibles, ce qui n'est pas rien, mais ce qui n'est pas encore une vie démocratique.

\section{Laïciser la laïcité}

Toutes la difficulté de cette question est d'éviter deux démesures : une laïcité purement antireligieuse et une laïcité soi-disant empirique, résultat d'un compromis momentané. Le philosophe au moins apporte cela : les faits seuls n'enseignent rien et on ne peut fonder un droit, une éthique et une politique sur les faits. Derrière toute conception prétendument empiriste, il y a une idée, parfois cachée, et cela est également très dangereux. Dès lors défendre une laïcité ouverte, apaisée, bienveillante, cela peut avoir un sens en communication politique, mais philosophiquement cela n'apporte rien, car c'est la laïcité elle-même qu'il faut défendre et cela en déterminant la juste place de la religion dans l'espace public et en montrant que la religion n'est pas un simple obscurantisme à dépasser, mais un élément constitutif de la culture. Cela conduit alors également à reconnaître que la laïcité n'a pas pour but d'isoler le citoyen dans la société et donc qu'elle n'a pas à remettre en cause les communautés du judaïsme, de l'Islam, du christianisme, etc. tant que celles-ci ne portent pas atteinte à la légitimité de l'Etat et à la dignité de la personne. Autrement dit, l'identité citoyenne et l'identité religieuse ne sont pas incompatibles et elles peuvent même coopérer l'une avec l'autre. La religion n'est pas une pure affaire privée comme voudrait le donner à penser un certain laïcisme de combat et son expression publique peut être profitable à la société. De ce point de vue la laïcité «à l'américaine », pour citer un pays dans lequel la liberté religieuse est première, n'est pas nécessairement mauvaise, même si elle est exposée à une autre dérive, celle de tout accepter, même au prix de ne pas protéger les citoyens. Tout cela pour dire qu'entre l'universalisme abstrait d'un citoyen pur de toute religion et le particularisme relativiste, il doit y avoir, il faut l'espérer, une autre voie. 
On voit au moins que pour concilier l'affirmation de la liberté religieuse, de sa manifestation, tout en luttant contre le fondamentalisme et les dérives sectaires, il est nécessaire que la réflexion sur la laïcité et celle sur la religion ne soient pas dissociées. C'est pourquoi il y a un travail permanent de laïcisation de la laïcité en acceptant la pluralité de ses figures historiques, en réinventant sans cesse sa concrétisation dans une situation, et en aidant la religion à être proprement religieuse, là aussi dans la diversité irréductible de ses formes. On a voulu défendre l'idée positive que l'Etat et la religion peuvent se développer de concert, si les Etats posent les conditions d'une dialogue et si les religions acceptent le dialogue entre elles et avec les Etats. Par suite, si la laïcité ne devient qu'un processus d'exclusion et de purification de toute foi religieuse, elle ne peut qu'échouer et augmenter ainsi le mal dans le monde. Il s'agit donc d'inventer une laïcité qui rassemble, et pas seulement d'une manière purement extérieure et contingente. Dès lors, la laïcité appartient à l'identité française, il est important de ne pas la figer dans sa figure historique actuelle, car au-delà de son appartenance à une histoire particulière, elle est aussi une idée de la séparation libre de toute facticité, et dans laquelle la religion demeure aussi l'avenir de l'humanité et n'est pas réduite à un passé à dépasser.

Il n'est donc pas possible de décréter a priori que telle religion est intégrable ou n'est pas intégrable dans la République, car cela dépend du travail de la République sur elle-même pour intégrer de nouvelles données culturelles et du travail de cette religion sur elle-même pour accepter le principe de la neutralité de l'Etat. Au moins pouvons-nous espérer que cette double réflexion puisse avoir lieu ; c'est un article de foi laïque ! Cela souligne encore une fois que l'Etat ne peut pas se contenter du rôle minimaliste de tenter de réguler les tensions spirituelles au coup par coup et qu'il doit prendre sa part dans ce processus de reconnaissance réciproque qui seul peut permettre d'éviter le choc des visions du monde.

\section{Religion et tolérance}

On a voulu montrer combien cette séparation de l'Eglise et de l'Etat ne va pas de soi, car il n'y a pas d'espace historique homogène, mais des traditions irréductibles qui doivent donc s'approprier de façon propre cette idée de laïcité, qui est l'idée d'un espace dans lequel religion et politique sont compossibles. Néanmoins il est possible d'approfondir encore la difficulté de la question en soulignant que le partage du religieux et du non religieux, qui semblait pourtant un point de départ stable, ne va nullement de soi. Comment penser la séparation si on ne parvient pas à distinguer clairement les éléments à séparer?

Emmanuel Levinas, très marqué par l'expérience du nihilisme en ce domaine, il cite à ce propos l'affaire Dreyfus, tente de montrer que « le judaïsme est à l'étroit dans le concept de religion tel que le formule la sociologie ${ }^{10} »$, dans la mesure où pour lui le judaïsme est une religion de tolérance car « la certitude de l'emprise de l'absolu sur l'homme - ou religion - ne se mue pas en expansion impérialiste qui dévore tous ceux qui la refuse ${ }^{11} »$. Dans son sens supérieur, inaccessible au laïcisme, la religion «brûle vers l'intérieur, comme une exigence infinie à l'égard de soi, comme une infinie responsabilité ${ }^{12} »$. Ainsi la laïcité n'est pas un slogan à brandir lors de chaque situation conflictuelle, mais l'occasion de réfléchir sur le sens d'une vraie religion et sur ce point Levinas cherche à écarter deux compréhensions erronées de la religion qui font qu'elle semble être un obstacle à la liberté. Il est essentiel de ne pas confondre religion et Eglise ; autrement dit la religion peut être autre chose qu'une institution et le danger commence quand elle se fige en institution, processus qui ne viendrait pas de la religion elle-même: «Le déclin des religions constituées en Eglises est un phénomène historique incontestable. Il ne tient pas à la méchanceté des hommes ; mais à l'avènement des Etats ${ }^{13}{ } \gg$. Comme

\footnotetext{
${ }^{10}$ Emmanuel Levinas, Difficile liberté, Le livre de proche, 2006, p. 370.

${ }^{11}$ Ibid., p. 262.

${ }^{12}$ Idem.

${ }^{13}$ Ibid. p. 324.
} 
on l'a vu, il est impossible de s'interroger sur la laïcité sans s'interroger sur l'essence de la religion et il apparaît maintenant que la religion n'est ni une institution, ni une forme de culture, ni un ensemble constitué de croyances, mais « une pulsation même de la vie où Dieu entre en rapport avec l'homme, et l'homme avec le monde ${ }^{14} \gg$. La seconde source de nihilisme selon Levinas se trouve dans la réduction de la religion à un culte privé qui repose souvent dans la trop paisible distinction du spirituel et du temporel. Ni le judaïsme ni le christianisme ne peuvent se comprendre comme un tête-à-tête avec Dieu : la vie religieuse, comme la vie éthique, se joue parmi les hommes, dans l'exercice d'une responsabilité, et ne relève donc pas de la seule vie privée. Cette distinction conceptuelle entre vie publique et vie privée ne peut donc pas être la réponse simple, trop simple, à la crise de la laïcité et pour aborder cette question il faut reconnaître que l'Etat et la religion, quand ils sont conformes à leur vérité, déploient deux sens de la justice, qu'ils sont deux façon de donner sens à l'être, à la vie.

Toutes ces analyses conceptuelles peuvent laisser sur leur faim ceux qui attendraient des solutions simples, un manuel de tolérance enseignant quelques recettes infaillibles pour ne pas rater l'unité de la société, mais le philosophe peut au moins montrer qu'il n'y a pas de technique de la paix sociale, qu'il s'agit d'une évolution historique lente liée à une prise de conscience, et qu'il ne faut pas enfermer la laïcité dans des oppositions trop brutales pour être attentif à tous ces glissements de sens de façon à penser d'une manière nouvelle la laïcité. Levinas définit ainsi la tâche du philosophe : « Les intellectuels en tant qu'intellectuels, quand ce sont de vrais intellectuels, ont pour mission de dégager et de mesurer les possibles que libèrent les glissements de sens annonçant des glissements de terrain, de montrer les présupposés d'une crevasse encore invisible à l'œil nu, souvent même d'une simple friabilité de fondation ${ }^{15} »$. L'opposition privé-public, religieux-non religieux, la confusion de l'Eglise et de la religion, etc. sont ces fissures presque invisibles dans la tolérance fondement de la paix.

\section{Conclusion}

On a voulu montrer qu'il n'est possible de répondre à la crise de la laïcité que par une séparation du religieux et du politique qui n'est pas une exclusion réciproque, mais une reconnaissance réciproque supposant qu'Etat et religion ne se comprennent plus comme des objets pétrifiés, car ce sont des processus. Il ne s'agit pas d'abolir le conflit entre Etat et religion qui n'est pas réductible, puisque cela engage deux sens de la justice, mais de le rendre viable, notamment avec l'étonnant développement des religions, par exemple en Chine. L'Etat ne se développe qu'en reconnaissant son autre que sont les religions, mais cette reconnaissance ne peut pas être unilatérale et demande aussi à ce que les religions comprennent quelle est leur propre place. On peut résumer ainsi les thèses engagées dans ce travail :

1. Il est essentiel d'assurer la neutralité de l'Etat pour la justice et pour la liberté religieuse, cette neutralité étant une condition a priori de la communauté politique. L'enjeu est de savoir comment réaliser cette neutralité dans chaque situation historique singulière.

2. La laïcité est un principe régulateur de la vie sociale qui peut prendre des formes historiques très différentes et qui doit être dans une constante mutation. Idéalisme et pragmatisme ne s'opposent pas.

3. La laïcité ne peut pas être une négation de la religion et elle doit reconnaître la place irréductible de cette dernière dans le processus de culture.

4. Elle n'est pas un simple compromis entre des forces en présence et interdit ce qui dans une religion peut être une violence faite aux personnes, toute atteinte à leur dignité de sujet libre et sujet de droit.

5. La laïcité est une réponse au nihilisme qui ne se substitue pas à la réponse religieuse, mais l'accompagne.

\footnotetext{
${ }^{14}$ Ibid. p. 284.

${ }^{15}$ Ibid. p. 422.
} 
6. La réflexion sur la laïcité est indissociable de la réflexion des religions sur elles-mêmes dans le cadre d'une reconnaissance réciproque entre le politique et le religieux.

7. La laïcité est une invitation pour la religion à ne pas se figer en simple institution, voire en force politique.

8. L'esprit des Lumières et l'obéissance religieuse ne sont pas incompatibles dans une identité en recherche.

9. La laïcité n'est pas un acquis, mais une tâche infinie d'élucidation à laquelle le philosophe participe. Ce n'est pas qu'un principe de limitation, mais également une source de créativité politique face à la nouveauté de chaque situation. On n'est fidèle à la laïcité qu'à la réinventer sans cesse.

La crise de la laïcité a donc une double face, car elle est à la fois la recherche d'un équilibre entre les Eglises comme institutions de manière à les limiter contre elles-mêmes d'une dérive en pouvoir politique et une interrogation sur le sens de l'expérience religieuse, en un sens très large, comme manière de donner sens à la vie. Ainsi l'athéisme, avec toutes les thèses qu'il contient sur l'homme, sur le monde et donc sur la vie elle-même, ne peut pas devenir une nouvelle doctrine d'Etat, car il serait aussi tyrannique que les religions d'Etat. Afin d'équilibrer les questions, ce qui est le rôle du philosophe, le danger de l'athéisme n'est pas moins grand quand il se comprend comme une indifférence au bien, un relativisme des valeurs, un individualisme absolu et finalement comme une vie solitaire guidée par la seule utilité. Tout cela pour souligner à nouveau que la pratique de la laïcité relève bien de la prudence, de cette sagesse pratique, qui n'est pas une simple habileté et qui travaille à la limitation des dérives de l'Etat et de la religion de manière à favoriser leur reconnaissance réciproque dans des circonstances toujours singulières et qu'il est impossible d'ignorer. 